\title{
Comportamento de Receptores em uma Rede de Frequência Única ISDB-T
}

\author{
Christian Fragoas Fernandez Rodrigues, Francisco de Assis Peres, Lisadro Lovisolo
}

\begin{abstract}
Multiple frequency networks were widely used for television broadcasting due to the inability of original television reception systems to demodulate interfered signals. With the advance of television broadcast technology to digital transmission using OFDM, distribution networks employ a smaller number of frequency channels than for analog broadcasting. In addition, single frequency networks become an important alternative, as they optimize spectrum usage, allowing contiguous regions to be covered using less frequency channels. Digital television receivers improvements make them more prepared to work in SFN scenarios, as they improve their capabilities to demodulate signals under adverse conditions. This work presents test results of digital television commercial receiver's behavior in a SFN. The tests evaluate and analyze their reception capabilities in a SFN scenario, at the receiver signals from two transmitters arrive in line of sight, this is done in several settings with different guard intervals. This laboratory test was applied to five digital television receptors. An improvement in the performance of receptors was observed in comparison to previous works. The impact of the guard interval configuration is also reported. The use of SFN generates a reception margin loss which is evaluated in this work. In addition, using a default receptor model we translate the power density values measured on laboratory to the minimal electrical field strength for reception.
\end{abstract}

Index Terms - OFDM. Digital television. Receptors performance in SFN.

\section{INTRODUÇÃO}

Com a atual escassez do espectro eletromagnético, $\checkmark$ radiodifusores e órgãos reguladores buscam otimizar a utilização do mesmo [1]. Redes de frequência única

Christian Fragoas Fernandez Rodrigues é Analista de Sistemas pelo Centro Universitário da Cidade e Estudante de Engenharia Elétrica com Ênfase em Telecomunicações pela Universidade do Estado do Rio de Janeiro, Rio de Janeiro, RJ, Brasil (e-mail: christianfragoas@gmail.com.br).

Francisco de Assisp Peres é Gerente do Departamento de Projetos e Instalações de Sistemas de Transmissão da TV Globo (e-mail: francisco.peres@tvglobo.com.br).

Lisandro Lovisolo, DSc, é Professor do Departamento de Eletrônica e Telecomunicações da Universidade do Estado do do Rio de Janeiro, Rio de Janeiro, RJ, Brasil (e-mail: lisandro@uerj.br).
(SFNs) tornam-se uma necessidade nesse cenário, pois nelas vários transmissores de um mesmo conteúdo utilizam a mesma frequência de transmissão. Em MFNs (Multi Frequency Networks) tradicionais, cada transmissor possui um canal de radiodifusão diferente em cada área de cobertura e nenhuma área adjacente utiliza o mesmo canal. Uma SFN possibilita a economia de espectro, pois cobre múltiplas regiões com um único canal de radiodifusão.

As MFNs foram muito utilizadas nos projetos de cobertura de televisão analógica, principalmente devido a limitações de receptores analógicos para corrigir dificuldades de propagação, como multipercursos. $\mathrm{O}$ plano básico de distribuição de canais elaborado pela Anatel restringe, no caso da televisão analógica, a utilização de um único canal de RF por área de cobertura.

Com o desenvolvimento da televisão digital o uso de uma rede de frequência única para o sinal de televisão digital passou a ser plausível. A melhoria nos receptores, que passaram a equalizar de maneira mais eficiente sinais sujeitos a multipercurso, permite a adoção de redes de frequência única.

Este trabalho visa determinar e analisar as condições limiares de recepção em visada direta, que é o pior caso, para projetos de radiodifusão de televisão digital quando utiliza-se de uma rede SFN, através de medidas feitas em laboratório simulando essas condições. Espera-se com este trabalho que o projeto de tais redes seja facilitado.

O projeto de uma SFN não é trivial. É necessário adequar a potência dos transmissores e a sincronia da rede para que todos os receptores de regiões que venham a receber sinais de diferentes estações transmissoras não sofram interferências dos sinais provenientes de diferentes transmissores[1]. Ou seja, é preciso garantir que sinais secundários sejam recebidos com atrasos dentro do intervalo de guarda do sinal principal. Por outro lado, caso um ou mais sinais secundários sejam recebidos fora do intervalo de guarda, as diferenças de potência relativa entre o sinal principal e os secundários devem ser tais que a interferência gerada pelos sinais secundários não impeça a recepção correta do sinal OFDM.

Como o uso de SFNs está cada vez mais comum, devido à 
economia de banda derivada de seu uso, o estudo das mesmas é cada vez mais necessário de forma a tornar as condições de análise e projeto de tais redes mais realistas e confiáveis.

Para que um receptor de TV digital funcione corretamente é necessário que ele não seja saturado pelos sinais incidentes e que obtenha sucesso na demodulação, mesmo com os efeitos de multipercurso. No caso de SFNs, temos que controlar estes efeitos uma vez que que os transmissores podem ser sincronizados. Isto é, podemos defasar os sinais dos diferentes transmissores de forma a evitar a interferência intersimbólica significativa, possibilitando uma demodulação do sinal OFDM.

\section{ESPECIFICAÇÃO DE TESTES E MEDIDAS DE DESEMPENHO DE} SFN

Os experimentos propostos permitem analisar os efeitos conjuntos da interferência intersimbólica e do desvanecimento por multipercurso em uma rede SFN com dois transmissores em visada direta. Inicialmente iremos analisar a constelação do símbolo OFDM na recepção, na presença dos dois sinais gerados pelos excitadores. Em seguida analisaremos o desempenho de receptores, capacidade de demodulação do sinal e a presença de blocos nas imagens reproduzidas pelos televisores na presença dos dois sinais e de ruído.

Em ambos os casos foram avaliadas diferentes condições pela variação dos parâmetros de atraso entre os sinais e diferença entre suas potências.

Os arranjos da Figura 1 e da Figura 2 foram concebidos de maneira a simular diferentes condições de recepção, atraso relativo e amplitudes de sinais recebidos em uma SFN. Isso permite obter medidas empíricas e assim avaliar o desempenho de receptores e o comportamento da constelação diante de diferentes condições de recepção. As configurações do sistema de transmissão utilizado são as apresentadas na Tabela 1.

TABELA 1

CONFIGURAÇÃO DO SISTEMA DE TV DIGITAL UTILIZADO NOS EXPERIMENTOS

\begin{tabular}{|c|c|}
\hline Sistema & SBTVD \\
\hline Canal & 29 \\
\hline Largura de Banda & $6 \mathrm{Mhz}$ \\
\hline Modulação Layer A & $64 \mathrm{QAM}$ \\
\hline FEC (foward error correction) & $3 / 4$ \\
\hline Intervalo de Guarda & $1 / 8(126 \mu \mathrm{s})$ \\
\hline Modo de Sincronismo & Atraso estático (offset) \\
\hline
\end{tabular}

A. Arranjo 1 - Comportamento da Recepção de Símbolos

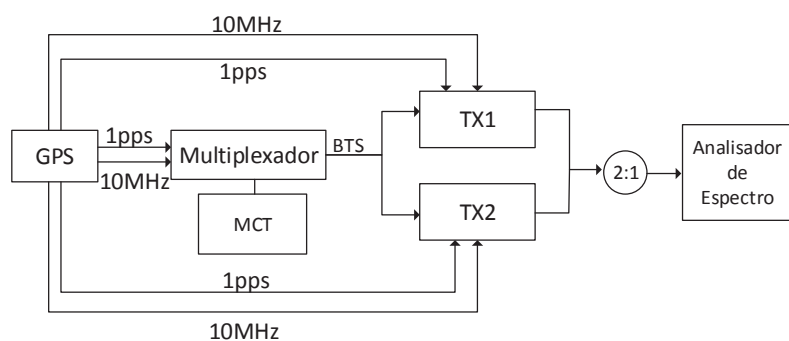

Figura 1. Diagrama da configuração utilizada em laboratório para analisar o comportamento dos símbolos na constelação na recepção.

O primeiro arranjo (Figura 1) foi concebido de maneira a analisar o comportamento da constelação (mapeamento de símbolos) recebida no receptor usando um TV analyzer. O arranjo é composto por um multiplexador alimentado pelas referências de tempo e frequência da rede (1pps, pulso por segundo, e $10 \mathrm{MHz}$ ) e pelo sinal ASI (Asynchronous Serial Interface) vindo do Mux Controller (MCT). É então gerado o BTS que alimenta os excitadores (Tx1 e TX2) que geram sinais de RF no canal 29 (560 a $566 \mathrm{MHz}$ ), que é analisado pelo TV analyzer.

O MCT é o responsável pelo sincronismo da rede. Ele compõe o quadro BTS com os bits de sincronismo e o atraso de cada transmissor na rede, enquanto os úlimos são responsáveis pela amplificação dos sinais na saída da rede para o receptor.

Com o TV analyzer foram realizadas capturas das constelações variando-se a diferença de nível entre portadoras de $0,5,10,15$ e $20 \mathrm{~dB}$ e de atraso relativo de 0 a $200 \mu \mathrm{s} \mathrm{em}$ passos de $20 \mu \mathrm{s}$.

\section{B. Arranjo 2 -Comportamento dos Receptores Comerciais de TV Digital}

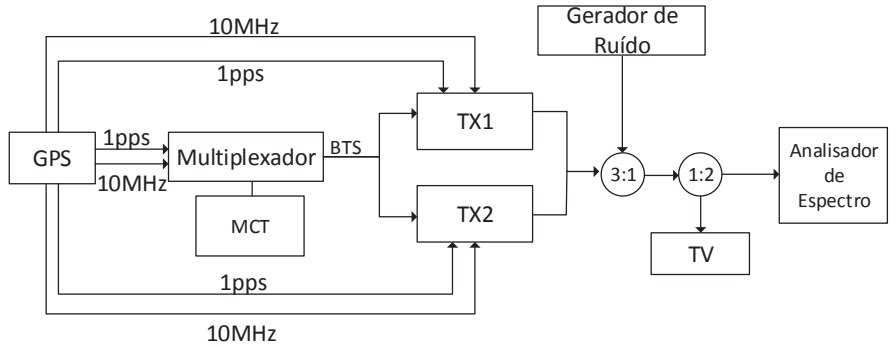

Figura 2. Diagrama da configuração utilizada em laboratório para medir o $\mathrm{C} / \mathrm{N}$ mínimo dos diversos televisores.

O arranjo da Figura 2 é similar ao da Figura 1 com o acréscimo do gerador de ruído ligado na saída de RF dos 
transmissores e do uso de um televisor como receptor. Isso permite analisar o comportamento do seu receptor embutido em condições similares às de uma recepção real com 2 transmissores em visada direta. Os transmissores foram configurados segundo a Tabela 1 .

Foram medidas as potências dos sinais de ambos os transmissores e do ruído branco adicionado com o auxílio de um analisador de espectro isso permite obter a razão sinalruído (SNR) mínima de recepção para cada teste. A SRN é definida como:

$$
S N R=\frac{\text { pot. sinal }}{\text { pot.ruído }}
$$

Variou-se a diferença de nível entre portadoras na faixa de 0 a $21 \mathrm{~dB}$ com passos de $3 \mathrm{~dB}$ e atrasos relativos de 30 a $120 \mu \mathrm{s}$, com passos de $30 \mu \mathrm{s}$, e de 120 a $200 \mu \mathrm{s}$, com passos de $10 \mu \mathrm{s}$. Para cada uma das configurações de atraso relativo e diferença de potência, mediu-se o SNR mínimo de recepção em cada um dos televisores disponíveis no laboratório a fim de comparar os diferentes televisores.

\section{Equipamentos utilizados}

Para implementar os arranjos ilustrados nas Figuras 1 e 2 foram utilizados os seguintes equipamentos:

1) Dois excitadores Linear recebem o sinal ASI do multiplexador com os sinais 1 pps e $10 \mathrm{MHz}$ a fim de gerar os dois sinais OFDM que irão compor a SFN;

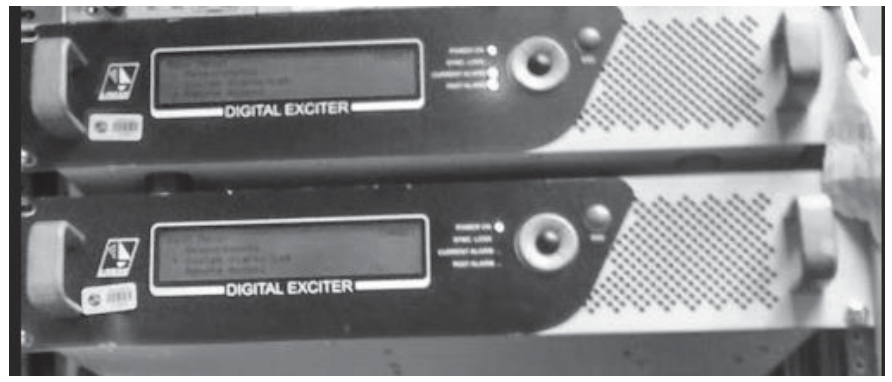

Figura 3. Excitadores Utilizados nos Experimentos.

2) Um GPS Epsilon Clock EC22S gerando os sinais de referência de tempo e frequência do sistema com o auxílio de uma antena de recepção de sinal GPS;

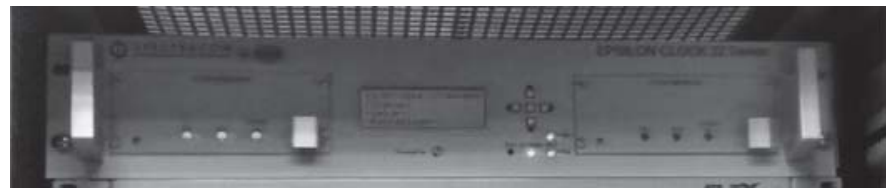

Figura 4. GPS Utilizado nos Experimentos.

3) Um multiplexador modelo NEC controlado pelo MCT (Mux Controller), que gera o sinal BTS que é transmitido pelos transmissores através de um TS obtido do seu controlador MCT;

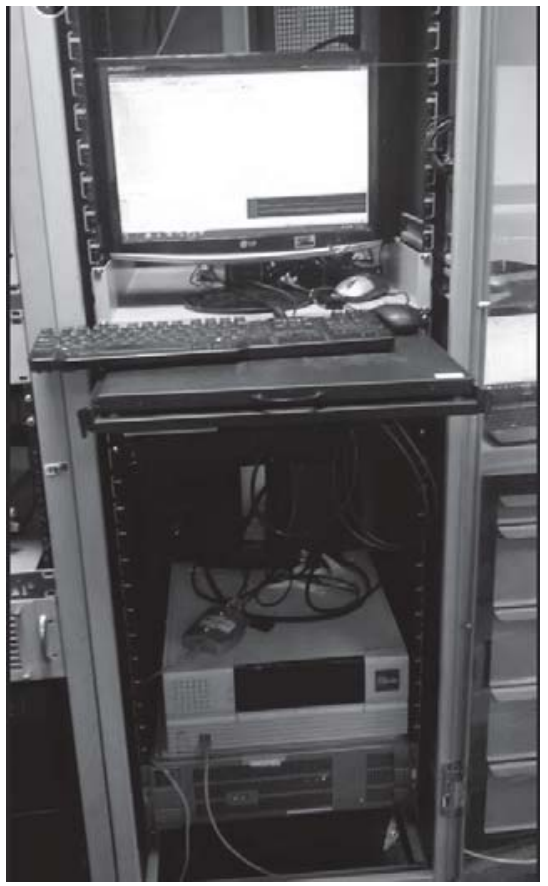

Figura 5. Mux e MCT Utilizados nos Experimentos.

4) Um transmissor de teste Rohde \& Schwarz SFE100 é utilizado para gerar o ruído branco em banda passante e simular as condições reais de transmissão;

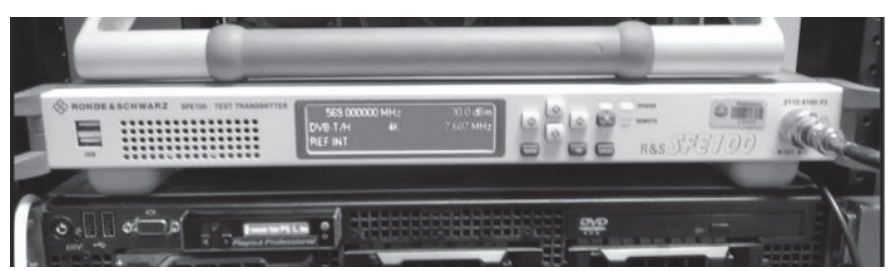

Figura 6. Transmissor de Teste Utilizado Como Gerador de Ruído nos Experimentos.

5) Uma série de televisores de diferentes fabricantes produzidos nos anos de 2012 e 2013 e um analisador de espectro Rohde \& Schawz ETH que irão receber os sinais OFDM e permite analisar os resultados.

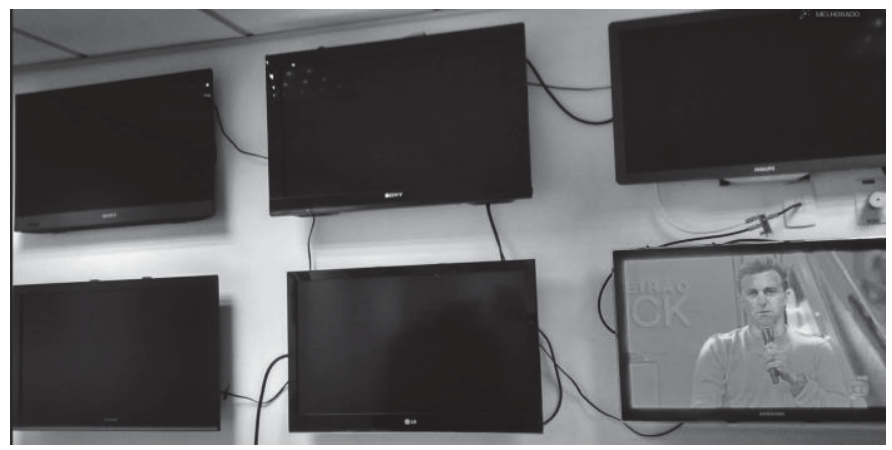

Figura 7. Conjunto de Televisores Utilizados nos Experimentos. 


\section{RESULTADOS E ANÁLISE}

A. Comportamento da Constelação do Receptor em SFN

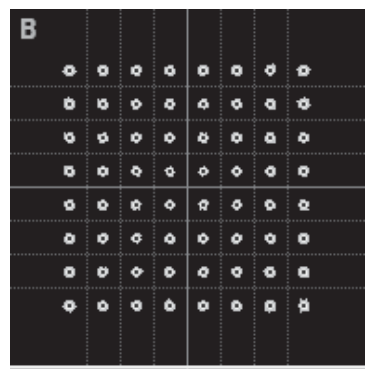

Figura 8. Constelação de um único sinal OFDM.

A Figura 8 representa a recepção no TV analyzer de um sinal OFDM da camada 2 com modulação 64QAM e suas 64 possíveis combinações de bits. Podem ser percebidos o espalhamento de amplitude e fase na recepção, devido às diversas distorções do canal.

As Figuras 9, 10, 11, 12 e 13 mostram o comportamento observado em laboratório da constelação de 64QAM (Layer B do sinal OFDM de televisão digital) das diferentes potências dos dois transmissores em função de diferentes atrasos relativos sem a presença de ruído.

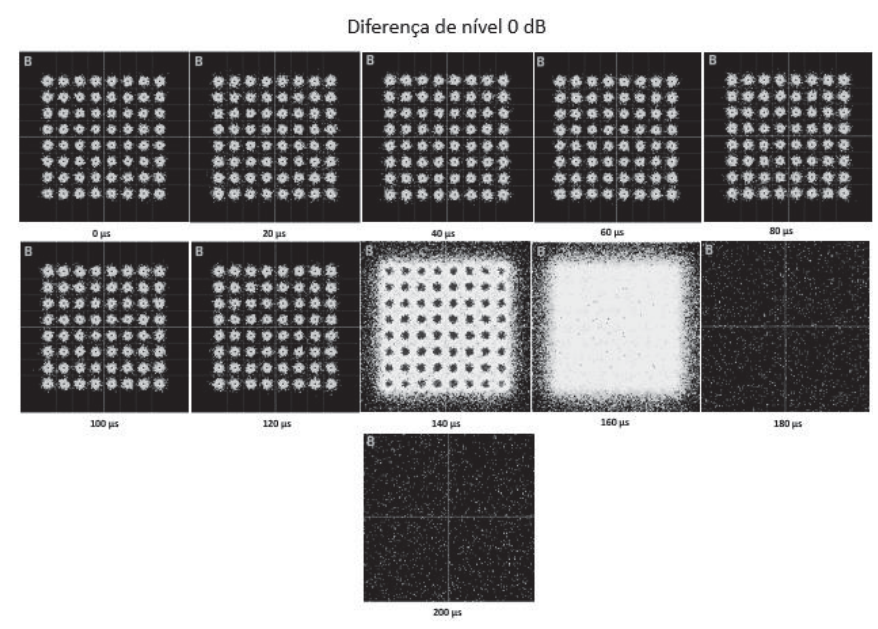

Figura 9. Comportamento da constelação com portadoras de níveis de potência iguais e com diferentes atrasos entre si.

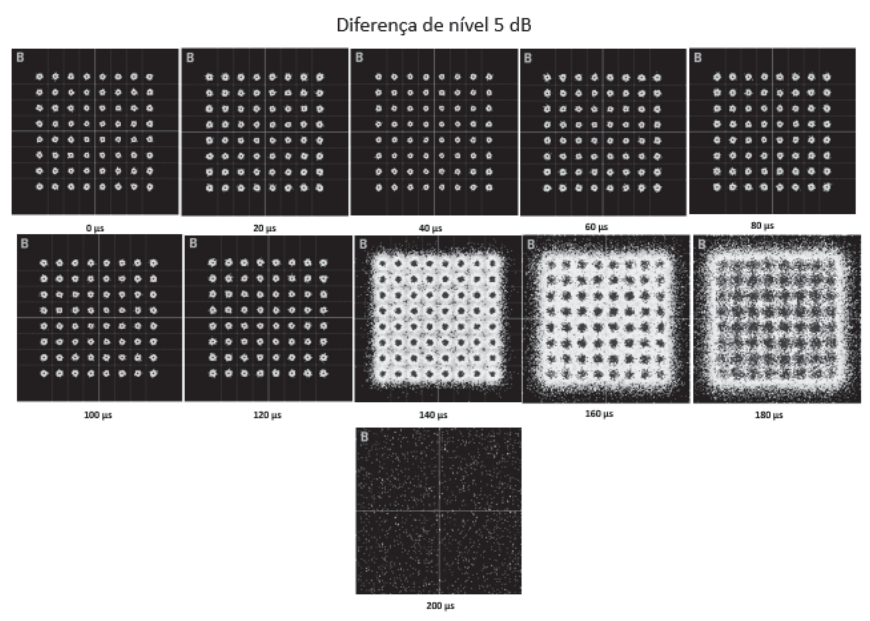

Figura 10. Comportamento da constelação com portadoras de níveis de potência divergentes em $5 \mathrm{~dB}$ e com diferentes atrasos entre si.

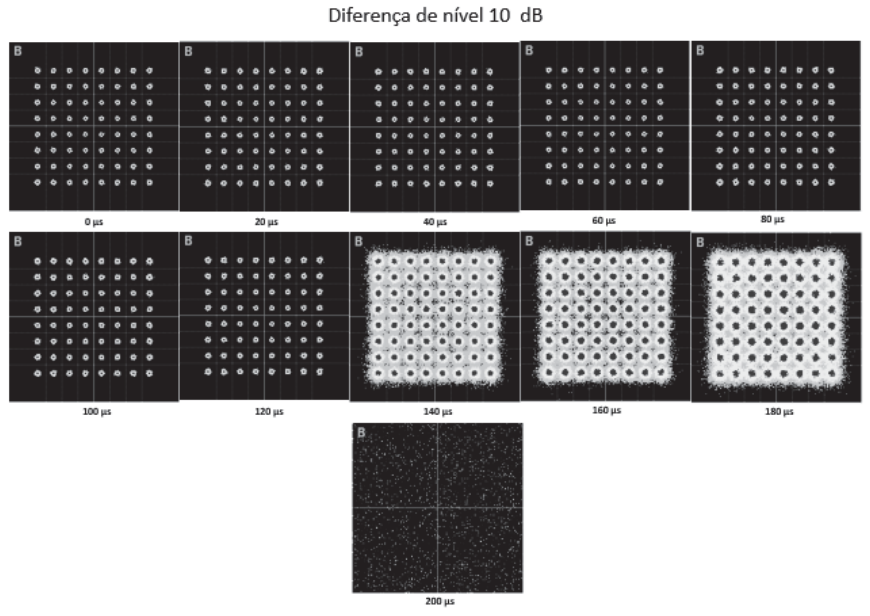

Figura 11. Comportamento da constelação com portadoras de níveis de potência divergentes em $10 \mathrm{~dB}$ e com diferentes atrasos entre si.

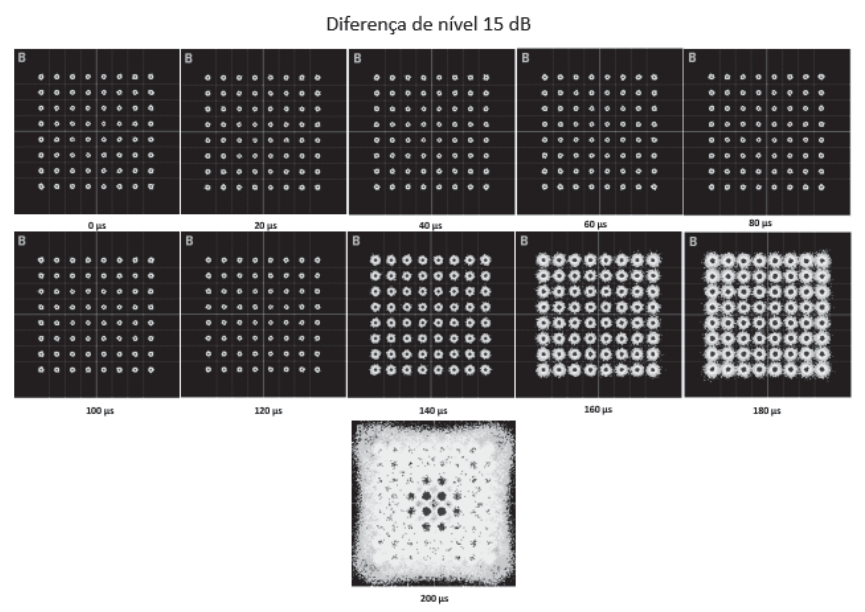

Figura 12. Comportamento da constelação com portadoras de níveis de potência divergentes em $15 \mathrm{~dB}$ e com diferentes atrasos entre si. 
Diferença de nível $20 \mathrm{~dB}$

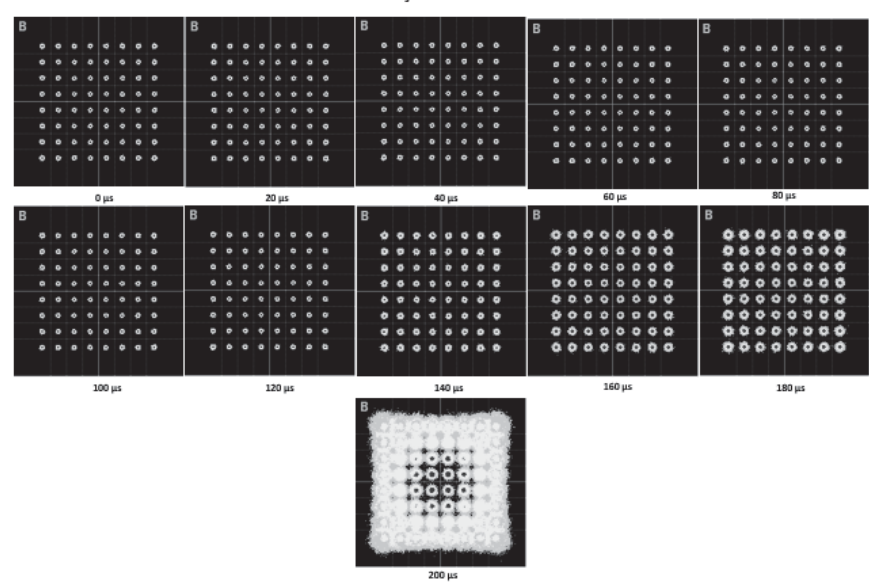

Figura 13. Comportamento da constelação com portadoras de níveis de potência divergentes em $20 \mathrm{~dB}$ e com diferentes atrasos entre si.

A partir das Figuras 9, 10, 11, 12 e 13 podemos constatar que a dispersão dos símbolos aumenta conforme aumentamos o atraso relativo para as diferentes SNR's entre os sinais de uma SFN. Observa-se também que quando o atraso ultrapassa o marco do intervalo de guarda de $126 \mu$ s, maior a dispersão devida à perda de ortogonalidade entre as portadoras OFDM. Combinando os resultados (atrasos e diferença entre potências), podemos constatar que a influência do sinal secundário pode ser menosprezada quando a diferença entre as potências dos transmissores for inferior a $15 \mathrm{~dB}$, condição na qual o limiar do intervalo de guarda passa a não ser tão relevante (mesmo com a perda da ortogonalidade entre as subportadoras). Isso ocorre pois nestes casos a interferência causada pelo sinal secundário é tão pequena que pode ser considerada insignificante em parte dos casos, não impactando a detecção correta dos símbolos. O comportamento observado em laboratório, ilustrado nas Figuras 9, 10, 11, 12 e 13, confirma os resultados descritos em [2].

A Figura 8 mostra os efeitos da recuperação de símbolos no receptor (observados em laboratório). Observa-se que a capacidade de recuperação dos símbolos é diferente em cada ponto da constelação. Isso é explicado na Figura 14, o espalhamento das portadoras é notadamente maior nas extremidades da constelação.

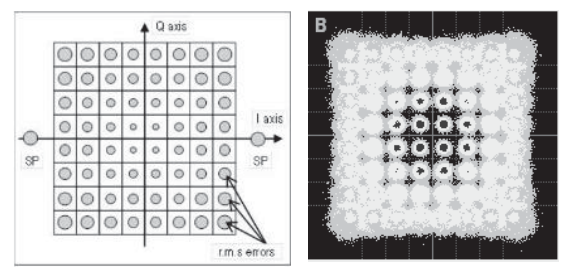

Figura 14. Comparação entre o estudo da ITU e o comportamento da constelação.
IV. RECEPÇÃO DO SINAL DE TV DIGITAL EM UMA REDE SFN COM 2 TRANSMISSORES SINCRONIZADOS

\section{A. Medidas Realizadas}

Foram realizados testes em laboratório com cinco televisores diferentes em uma SFN com dois transmissores conforme o diagrama da Figura 2. Mediu-se a potência mínima necessária para a correta recepção do sinal OFDM para cada televisor apresentado na Tabela 2. Para uma potência de sinal recebida de $-40 \mathrm{dBm}$ variou-se a potência do gerador de ruído e calculou-se o $\mathrm{C} / \mathrm{N}$ mínimo para a recepção com apenas um transmissor (Tabela 2). A terceira etapa foi medir o C/N mínimo nas diversas condições de recepção em um SFN de dois transmissores em SFN. Manteve-se a potência de transmissão de um dos transmissores em $-40 \mathrm{dBm}$ enquanto variou-se a potência do segundo transmissor a fim de se obter a diferença de nível,

$$
\begin{gathered}
\text { Dif. Nível }(d B)=\text { Pot. Tx1 }(d B m)-\text { Pot.Tx2 }(d B m)= \\
-40 d B m-P o t . T \times 2(d B m) .
\end{gathered}
$$

Caracterizou-se nas diferentes condições de recepção a potência de ruído do gerador de ruído, para que se calculasse a C/N mínima de recepção:

$$
C / N_{\min }=\frac{\operatorname{Pot}_{T x 1+T x 2}}{N_{\max }}
$$


TABELA 2

C/N MÍNIMO E POTÊNCIA MÍNIMA PARA A CORRETA RECEPÇÃO DOS SÍMBOLOS MEDIDOS EM LABORATÓRIO

\begin{tabular}{|c|c|c|}
\hline Televisores testados & $\mathrm{C} / \mathrm{N}_{\text {minimo }}(\mathrm{dB})$ & $\begin{array}{c}\text { Potência mínima de } \\
\text { recepção }(\mathrm{dBm})\end{array}$ \\
\hline A & 17,8 & $-80,6$ \\
\hline B & 17,8 & $-81,1$ \\
\hline C & 16,7 & $-83,2$ \\
\hline D & 16,7 & $-82,2$ \\
\hline E & 16,7 & $-84,8$ \\
\hline
\end{tabular}

TABELA 3

C/N MÍNIMO MEDIDOS NO TELEVISOR A

\begin{tabular}{|c|c|c|c|c|c|c|c|c|}
\hline \multicolumn{7}{|c|}{ C/N min para diferentes configurações de diferença de nível e atraso } \\
\hline & \multicolumn{7}{|c|}{ Atraso relativo entre portadoras ( $\boldsymbol{~}$ s) (IG 126 $\boldsymbol{\mu s})$} \\
\hline $\begin{array}{c}\text { Dif } \\
\text { Nível(dB) }\end{array}$ & $\mathbf{3 0}$ & $\mathbf{6 0}$ & $\mathbf{9 0}$ & $\mathbf{1 2 0}$ & $\mathbf{1 3 0}$ & $\mathbf{1 5 0}$ & $\mathbf{1 7 0}$ & $\mathbf{2 0 0}$ \\
\hline $\mathbf{0}$ & 23 & 25,5 & 24,7 & 24 & $\mathrm{x}$ & $\mathrm{x}$ & $\mathrm{x}$ & $\mathrm{x}$ \\
\hline $\mathbf{3}$ & 20,6 & 21,6 & 20,6 & 20,6 & $\mathrm{x}$ & $\mathrm{x}$ & $\mathrm{x}$ & $\mathrm{x}$ \\
\hline $\mathbf{6}$ & 18,8 & 20,8 & 19,8 & 19,8 & $\mathrm{x}$ & $\mathrm{x}$ & $\mathrm{x}$ & $\mathrm{x}$ \\
\hline $\mathbf{9}$ & 18,2 & 18,2 & 18,2 & 18,2 & 23,9 & $\mathrm{x}$ & 21,2 & 25,7 \\
\hline $\mathbf{1 2}$ & 17,9 & 17,9 & 17,9 & 17,9 & 18,9 & 19,9 & 19,9 & 20,9 \\
\hline $\mathbf{1 5}$ & 17,8 & 17,8 & 17,9 & 17,8 & 18,8 & 19,8 & 19,8 & 20,8 \\
\hline $\mathbf{1 8}$ & 17,8 & 17,8 & 17,8 & 17,8 & 17,8 & 19,8 & 19,8 & 20,8 \\
\hline $\mathbf{2 1}$ & 17,8 & 17,8 & 17,8 & 17,8 & 17,8 & 17,8 & 17,8 & 18,8 \\
\hline
\end{tabular}

TABELA 4

C/N MÍNIMO MEDIDO NO TELEVISOR B

\begin{tabular}{|c|c|c|c|c|c|c|c|c|}
\hline \multicolumn{7}{|c|}{ C/N min para diferentes configurações de diferença de nível e atraso } \\
entre portadoras (dB) \\
\hline $\begin{array}{c}\text { Dif } \\
\text { Nível(dB) }\end{array}$ & $\mathbf{3 0}$ & $\mathbf{6 0}$ & $\mathbf{9 0}$ & $\mathbf{1 2 0}$ & $\mathbf{1 3 0}$ & $\mathbf{1 5 0}$ & $\mathbf{1 7 0}$ & $\mathbf{2 0 0}$ \\
\hline $\mathbf{0}$ & 23 & 23 & 23 & 23 & $\mathrm{x}$ & $\mathrm{x}$ & $\mathrm{x}$ & $\mathrm{x}$ \\
\hline $\mathbf{3}$ & 20,6 & 20,6 & 21,6 & 21,6 & 21,6 & 21,6 & 21,6 & 21,6 \\
\hline $\mathbf{6}$ & 19,8 & 19,8 & 19,8 & 19,8 & 19,8 & 19,8 & 19,8 & 19,8 \\
\hline $\mathbf{9}$ & 18,2 & 18,2 & 18,2 & 18,2 & 18,2 & 19,2 & 19,2 & 19,2 \\
\hline $\mathbf{1 2}$ & 17,9 & 17,9 & 17,9 & 17,9 & 17,9 & 17,9 & 17,9 & 17,9 \\
\hline $\mathbf{1 5}$ & 17,8 & 17,8 & 17,8 & 17,8 & 17,8 & 17,8 & 17,8 & 17,8 \\
\hline $\mathbf{1 8}$ & 17,8 & 17,8 & 17,8 & 17,8 & 17,8 & 17,8 & 17,8 & 17,8 \\
\hline $\mathbf{2 1}$ & 17,8 & 17,8 & 17,8 & 17,8 & 17,8 & 17,8 & 17,8 & 17,8 \\
\hline
\end{tabular}

TABELA 5

C/N MÍNIMO MEDIDO NO TELEVISOR C

\begin{tabular}{|c|c|c|c|c|c|c|c|c|}
\hline \multicolumn{7}{|c|}{ C/N min para diferentes configurações de diferença de nível e atraso } \\
entre portadoras (dB) \\
\hline $\begin{array}{c}\text { Dif } \\
\text { Nível(dB) }\end{array}$ & $\mathbf{3 0}$ & $\mathbf{6 0}$ & $\mathbf{9 0}$ & $\mathbf{1 2 0}$ & $\mathbf{1 3 0}$ & $\mathbf{1 5 0}$ & $\mathbf{1 7 0}$ & $\mathbf{2 0 0}$ \\
\hline $\mathbf{0}$ & 23 & 23 & 23 & 23 & $\mathrm{x}$ & $\mathrm{x}$ & $\mathrm{x}$ & $\mathrm{x}$ \\
\hline $\mathbf{3}$ & 20,6 & 20,6 & 20,6 & 20,6 & 20,6 & 20,6 & 21,6 & 21,6 \\
\hline $\mathbf{6}$ & 18,8 & 18,8 & 18,8 & 18,8 & 18,8 & 18,8 & 19,8 & 19,8 \\
\hline $\mathbf{9}$ & 18,2 & 18,2 & 18,2 & 18,2 & 18,2 & 18,2 & 18,2 & 18,2 \\
\hline $\mathbf{1 2}$ & 17,9 & 17,9 & 17,9 & 17,9 & 17,9 & 17,9 & 17,9 & 17,9 \\
\hline $\mathbf{1 5}$ & 16,7 & 16,7 & 17,8 & 17,8 & 17,8 & 17,8 & 17,8 & 17,8 \\
\hline $\mathbf{1 8}$ & 16,7 & 16,7 & 17,8 & 17,8 & 17,8 & 17,8 & 17,8 & 17,8 \\
\hline $\mathbf{2 1}$ & 16,7 & 16,7 & 16,7 & 16,7 & 16,7 & 16,7 & 17,8 & 17,8 \\
\hline
\end{tabular}

TABELA 6

C/N MÍNIMO MEDIDO NO TELEVISOR D

\begin{tabular}{|c|c|c|c|c|c|c|c|c|}
\hline \multicolumn{7}{|c|}{ C/N min para diferentes configurações de diferença de nível e atraso } \\
entre portadoras (dB) \\
\hline $\begin{array}{c}\text { Dif } \\
\text { Nível(dB) }\end{array}$ & $\mathbf{3 0}$ & $\mathbf{6 0}$ & $\mathbf{9 0}$ & $\mathbf{1 2 0}$ & $\mathbf{1 3 0}$ & $\mathbf{1 5 0}$ & $\mathbf{1 7 0}$ & $\mathbf{2 0 0}$ \\
\hline $\mathbf{0}$ & 22 & 22 & 22 & 23 & $\mathrm{x}$ & $\mathrm{x}$ & $\mathrm{x}$ & $\mathrm{x}$ \\
\hline $\mathbf{3}$ & 19,6 & 19,6 & 19,6 & 19,6 & 20,6 & 20,6 & 20,6 & 20,6 \\
\hline $\mathbf{6}$ & 18,8 & 18,8 & 18,8 & 18,8 & 19,8 & 19,8 & 19,8 & 19,8 \\
\hline $\mathbf{9}$ & 18,2 & 18,2 & 18,2 & 18,2 & 19,2 & 19,2 & 19,2 & 19,2 \\
\hline $\mathbf{1 2}$ & 17,9 & 17,9 & 17,9 & 17,9 & 17,9 & 17,9 & 17,9 & 17,9 \\
\hline $\mathbf{1 5}$ & 17,8 & 17,8 & 17,8 & 17,8 & 17,8 & 17,8 & 17,8 & 17,8 \\
\hline $\mathbf{1 8}$ & 17,8 & 17,8 & 17,8 & 17,8 & 16,7 & 16,7 & 16,7 & 17,8 \\
\hline $\mathbf{2 1}$ & 17,8 & 16,7 & 17,8 & 16,7 & 16,7 & 16,7 & 16,7 & 16,7 \\
\hline
\end{tabular}


TABELA 7

C/N MÍNIMO MEDIDO NO TELEVISOR E

\begin{tabular}{|c|c|c|c|c|c|c|c|c|}
\hline \multicolumn{7}{|c|}{ C/N min para diferentes configurações de diferença de nível e atraso } \\
entre portadoras (dB) \\
\hline & \multicolumn{7}{|c|}{ Atraso relativo entre portadoras ( $\boldsymbol{\mu s )}(\mathbf{I G} \mathbf{1 2 6} \boldsymbol{\mu s})$} \\
\hline $\begin{array}{c}\text { Dif } \\
\text { Nível(dB) }\end{array}$ & $\mathbf{3 0}$ & $\mathbf{6 0}$ & $\mathbf{9 0}$ & $\mathbf{1 2 0}$ & $\mathbf{1 3 0}$ & $\mathbf{1 5 0}$ & $\mathbf{1 7 0}$ & $\mathbf{2 0 0}$ \\
\hline $\mathbf{0}$ & 22 & 22 & 22 & 22 & 25,5 & $\mathrm{X}$ & $\mathrm{X}$ & $\mathrm{X}$ \\
\hline $\mathbf{3}$ & 19,6 & 19,6 & 19,6 & 20,6 & 21,6 & 24,1 & 23,9 & 24,9 \\
\hline $\mathbf{6}$ & 18,8 & 18,8 & 18,8 & 19,8 & 19,8 & 19,8 & 19,8 & 19,8 \\
\hline $\mathbf{9}$ & 18,2 & 18,2 & 18,2 & 18,2 & 18,2 & 18,2 & 18,2 & 18,2 \\
\hline $\mathbf{1 2}$ & 17,9 & 17,9 & 17,9 & 17,9 & 17,9 & 17,9 & 17,9 & 17,9 \\
\hline $\mathbf{1 5}$ & 16,7 & 16,7 & 17,8 & 17,8 & 17,8 & 17,8 & 17,8 & 17,8 \\
\hline $\mathbf{1 8}$ & 16,7 & 16,7 & 17,8 & 17,8 & 17,8 & 17,8 & 17,8 & 17,8 \\
\hline $\mathbf{2 1}$ & 16,7 & 16,7 & 17,8 & 17,8 & 17,8 & 17,8 & 17,8 & 17,8 \\
\hline
\end{tabular}

\section{B. Perda de Margem devido à SFN}

A Tabela 8 mostra a perda de margem no pior caso, conforme calculada na Equação 4 de um receptor comercial atual.

$$
\text { Perda de } \operatorname{margem}(d B)=C / N_{\text {min.1Tx }}(d B)-C / N_{\text {min.SFN }}(d B)
$$

TABELA 8

PERDA DE MARGEM DE C/N EM UMA SFN PARA DIVERSOS TELEVISORES

\begin{tabular}{|c|c|c|}
\hline Modelo & Dentro do IG & $\begin{array}{c}\text { Fora do } \\
\text { IG }\end{array}$ \\
\hline A & 5 & 9 \\
\hline B & 5 & 3 \\
\hline C & 6 & 4 \\
\hline D & 4 & 4 \\
\hline E & 5 & 7 \\
\hline
\end{tabular}

A partir da média simples dos valores da Tabela 8, calculamos a perda de margem média em um SFN para receptores comerciais (Tabela 9).

TABELA 9

PERDA DE MARGEM DE C/N DEVIDO A PRESENÇA DE UM SEGUNDO SINAL EM UMA REDE SFN

\begin{tabular}{|c|c|}
\hline \multicolumn{2}{|c|}{ Perda de margem de C/N (dB) } \\
\hline & Receptor comercial \\
\hline Dentro do IG & 5 \\
\hline Fora do IG & 5,4 \\
\hline
\end{tabular}

Em todos os casos não foi possível demodular o sinal, com exceção do televisor $\mathrm{E}$, após o intervalo de guarda $(126 \mu \mathrm{s})$ com os sinais dos transmissores com diferença de nível de
0dB. Porém, em todos os demais casos, com exceção do televisor A, foi possível a demodulação nas demais condições mesmo que com um aumento da razão sinal-ruído mínima necessária. O que demonstra as diferentes máscaras de recepção como em [2].

\section{CAMPO MÍNIMO PARA RECEPÇÃO EM SFN}

\section{A. Modelo de cálculo}

Para o cálculo da intensidade mínima de campo elétrico que chega as antenas para a recepção, considerando apenas antenas externa localizada em uma região de SFN. Os fatores apontados na Tabela 10 são em parte referentes as condições de recepção externas (após o receptor) consideradas no Planejamento de Canais de TV Digital do CPqD [3].

TABELA 10

FATORES UTILIZADOS PARA O CÁLCULO DO CAMPO MÍNIMO UTILIZANDO ANTENA EXTERNA

\begin{tabular}{|c|c|c|}
\hline Fator & Símbolo & Valor \\
\hline Largura de Faixa (MHz) & B & 6 \\
\hline $\begin{array}{l}\text { Constante de Boltzmann } \\
(\mathrm{Ws} / \mathrm{K})\end{array}$ & K & $1,38 \mathrm{E}-23$ \\
\hline Temperatura absoluta (K) & $\mathrm{T}$ & 290 \\
\hline Ruído térmico $(\mathrm{dBm})$ & $\mathrm{Nt}$ & $-106,20$ \\
\hline Frequência central (MHz) & $\mathrm{fb}$ & 563 \\
\hline Comprimento de onda $(\mathrm{m})$ & 1 & 0,53 \\
\hline $\begin{array}{l}\text { Área efetiva da antena } \\
\text { isotrópica }\left(\mathrm{dBm}^{2}\right)\end{array}$ & $\mathrm{Ai}$ & $-16,46$ \\
\hline $\begin{array}{l}\text { Ganho do dipolo de meia onda em relação à antena } \\
\text { isotrópica }(\mathrm{dBi})\end{array}$ & Gi & 2,15 \\
\hline $\begin{array}{l}\text { Ganho da antena em relação } \\
\text { ao dipolo de meia-onda (dBd) }\end{array}$ & G & 10 \\
\hline Área Efetiva da Antena (dBm2) & A & $-6,31$ \\
\hline Impedância intrínseca $(\Omega)$ & $\mathrm{h}$ & 377 \\
\hline $\begin{array}{l}\text { Fator de dipolo } \\
(\mathrm{dBm}-\mathrm{dB} \mu \mathrm{V} / \mathrm{m})\end{array}$ & $\mathrm{Kd}$ & $-130,07$ \\
\hline Perda em cabos $(\mathrm{dB})$ & $\mathrm{Lf}$ & 4 \\
\hline $\begin{array}{l}\text { Margem frente a ruído } \\
\text { produzido pelo homem }(\mathrm{dB})\end{array}$ & $\mathrm{Mm}$ & 0 \\
\hline
\end{tabular}

Baseando-se nos valores das Tabelas 3, 4, 5, 6 e 7 foram medidos os valores de $\mathrm{C} / \mathrm{N}$ mínimos, a figura de ruído do receptor, a margem de multipercurso em SFN, e, com a Equação 5, foi calculada a mínima potência do sinal (Tabela 11).

$$
\begin{gathered}
P_{S}(d B m)=N_{t}(d B m)+F_{r}(d B m)+C / N(d B) \\
+M_{S F N}(d B)
\end{gathered}
$$


TABELA 11

ESPECIFICAÇÕES MEDIDAS PARA OS RESPECTIVOS RECEPTORES DE TV DIGITAL

\begin{tabular}{|c|c|c|c|c|}
\hline \multirow{2}{*}{ Receptor } & $\begin{array}{c}\text { Limiar } \\
\text { de C/N } \\
\text { (dB) do } \\
\text { sistema } \\
\text { digital }\end{array}$ & $\begin{array}{c}\text { Figura } \\
\text { de ruído } \\
\text { do } \\
\text { receptor } \\
\text { (dB) }\end{array}$ & $\begin{array}{c}\text { Margem de } \\
\text { Multipercurso } \\
\text { em SFN (dB) }\end{array}$ & $\begin{array}{c}\text { Mínima } \\
\text { potência } \\
\text { do sinal } \\
\text { (dBm) }\end{array}$ \\
\cline { 2 - 5 } & \multicolumn{4}{|c|}{ Símbolo } \\
\cline { 2 - 5 } & $\mathbf{C} / \mathbf{N}$ & $\mathbf{F r}$ & Msfn & Ps \\
\hline $\mathrm{A}$ & 17,8 & 7,8 & 5,2 & $-75,40$ \\
\hline $\mathrm{B}$ & 17,8 & 7,3 & 5,2 & $-75,90$ \\
\hline $\mathrm{C}$ & 16,7 & 6,3 & 6,3 & $-76,90$ \\
\hline $\mathrm{D}$ & 16,7 & 6,3 & 4,2 & $-79,00$ \\
\hline $\mathrm{E}$ & 16,7 & 4,7 & 5,3 & $-79,50$ \\
\hline
\end{tabular}

\section{B. Resultados de campo mínimo em SFN}

Agora, considerando o sistema de recepção como especificado na Tabela 9, podemos calcular a mínima intensidade de campo na antena de recepção[4]

$$
\begin{aligned}
E_{\text {min }}(d B \mu V / m) & =P_{s}(d B m)+L_{f}(d B)+M_{m}(d B) \\
& -G(d B)-k_{d}(d B m-d B \mu V / m)
\end{aligned}
$$

TABELA 12

INTENSIDADE DE CAMPO MÍNIMA PARA DIVERSOS RECEPTORES DE TV DIGITAL

\begin{tabular}{|c|c|}
\hline Receptor & $\begin{array}{c}\text { Intensidade de Campo Mínima } \\
\left(\mathbf{E}_{\mathbf{m i n}} \mathbf{( \mathbf { d B m } )}\right.\end{array}$ \\
\hline $\mathrm{A}$ & 48,68 \\
\hline $\mathrm{B}$ & 48,18 \\
\hline $\mathrm{C}$ & 47,18 \\
\hline $\mathrm{D}$ & 45,08 \\
\hline $\mathrm{E}$ & 44,58 \\
\hline
\end{tabular}

\section{Fator de correção para localidades}

Para o atendimento de pelo menos $90 \%$ do tempo, da curva de propagação apresentada na Figura 15 , para $95 \%$ das localidades, é necessária uma correção $(\Delta L)$ com um fator de distribuição $(\mu)$ de 1.64 vezes do desvio padrão em grande escala $\left(\sigma_{m}\right)$, já que consideramos um grande número de elementos, de 5,5dB [3]. O resultado da correção da curva normal em decibéis é

$$
\Delta L(d B)=\mu \cdot \sigma m(d B)
$$

Foi adotado o fator de correção da curva para 95\% das localidades, pois é a correção utilizada na ITU-R BT. 1368-12 [3]. A correção de $95 \%$, ao contrário de $70 \%$ adotado em [4], é a mais adequada para TV digital, visto que o não atendimento dos valores limiares aqui especificados significa a não demodulação do sinal transmitido.

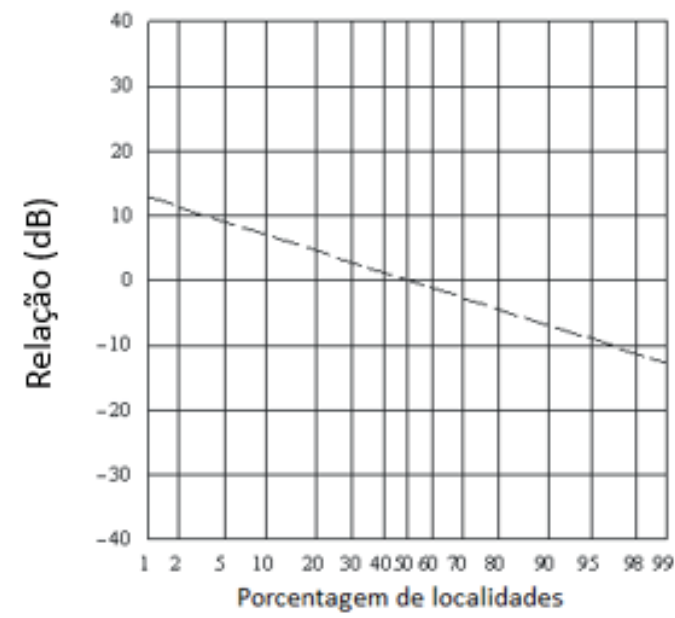

Figura 15. Relação de campo para uma dada porcentagem de localidades para um campo para $50 \%$ de localidades.

O campo mínimo corrigido pode ser obtido

$$
E_{\text {corrigido }}(d B \mu V / m)=E_{\min }(d B \mu V / m)+\Delta L(d B)
$$

O que nos dá a Tabela 12, com os respectivos valores de campo mínimo de planejamento.

TABELA 13

CAMPO MÍNIMO DE PLANEJAMENTO PROPOSTO PARA OS DIVERSOS RECEPTORES DE TV DIGITAL

\begin{tabular}{|c|c|}
\hline Receptor & $\begin{array}{c}\text { Campo mínimo de planejamento } \\
(\boldsymbol{d} \boldsymbol{B} \boldsymbol{\mu} \boldsymbol{V} / \boldsymbol{m})\end{array}$ \\
\hline Samsung UN32D5500RGXZD & 57,7 \\
\hline Philips 32PFL7606D/78 & 57,2 \\
\hline LG 32CS460 & 56,2 \\
\hline Sony KDL-32EX525 & 54,1 \\
\hline Panasonic TC-L32X30B & 53,6 \\
\hline
\end{tabular}

O pior caso apresentado na Tabela 13 estabelece um novo patamar de campo mínimo de $58 \mathrm{~dB} \mu \mathrm{V} / \mathrm{m}$. Assim, os valores de campo de planejamento necessários para um correto projeto de TV digital visando o cenário atual, é $7 \mathrm{~dB}$ maior que o campo mínimo do planejamento de $51 \mathrm{~dB} \mu \mathrm{V} / \mathrm{m}[4]$.

\section{CONCLUSÕES}

Os resultados obtidos demonstram não só a viabilidade de SFNs, como a evolução dos receptores de TV digital, porém com uma leve variação de desempenho entre eles. Vê-se que talvez seja necessário estabelecer um novo valor mínimo para o campo elétrico em projetos de difusão de TV digital. Quando comparam-se os resultados apresentados aqui aos apresentados em [4], verifica-se a necessidade de um incremento de $7 \mathrm{~dB}$ no valor padrão de campo elétrico mínimo utilizado em projetos de $51 \mathrm{~dB} \mu \mathrm{V} / \mathrm{m}$. Isto é necessário, no projeto de cobertura de transmissores de TV Digital, pois 
SFNs têm se tronado cada vez mais comuns. O valor de campo mínimo de planejamento de $58 \mathrm{~dB} \mu \mathrm{V} / \mathrm{m}$ obtido em nossa análise, contempla a perda de margem devido à SFN, e o fator de correção de $95 \%$ (conforme padrões internacionais de planejamento de TV digital).

\section{REFERÊNCIAS}

[1] Duca, Carolina, Yamada, Fujio. "Distribuição de sinais para o sistema Brasileiro de TV Digital”, Revista de Radiodifusão - SET, vol. 3, 2009.

[2] International Telecommunications Union, "Report ITU-R BT.2209-1:

Calculation model for SFN reception and reference receiver characteristics of ISDB-T system", Geneva, 2013.

[3] International Telecommunication Union, "Report ITU-R BT.1368-12: Planing criteria, including protection ratios, for digital terrestrial television services in the VHF/UHF bands," Geneva, 2015.

[4] Fundação CPqD - Centro de Pesquisa e Desenvolvimento em

Telecomunicações. "Planejamento de Canais de TV Digital", São Paulo, 2003.

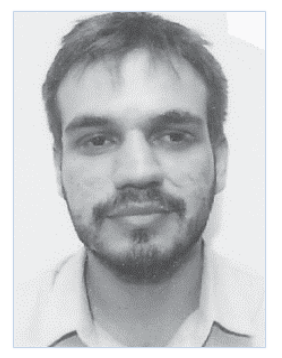

Chrisitan Fragoas Fernandez Rodrigues, estudante de Engenharia Elétrica com Ênfase em Telecomunicações pela Universidade do Estado do Rio de Janeiro, formado em Análise e Desenvolvimento de Sistemas pelo Centro Universitário da Cidade (2011). Atualmente é estagiário da TV Globo, na área de projetos de Transmissão de TV Digital.

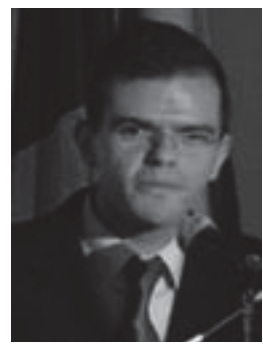

Francisco de Assis Peres graduou-se em Engenharia de Telecomunicações pela Universidade Federal Fluminense, conclui o mestrado em Eletromagnetismo pela Pontifícea Universidade Católica do Rio de Janeiro. Trabalhou por 12 anos como engenheiro de projetos na TV Globo desenhando antenas, sistemas distribuídos para sistemas MFN e SFN. Atualmente gerencia o departamento de projetos e instalações de sistemas de transmissão.

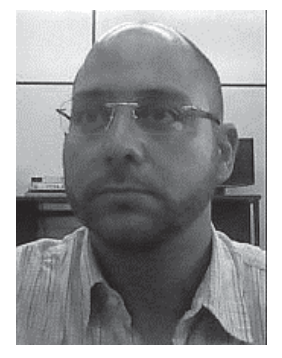

Lisandro Lovisolo concluiu o doutorado em Engenharia Elétrica pela Universidade Federal do Rio de Janeiro em 2006. Atualmente é Professor do Departamento de Eletrônica e Telecomunicações da Universidade do Estado do Rio de Janeiro. Além disso participa do Programa de Pós-Graduação em Engenharia Eletrônica dessa instituição. Fundou o PROSAICO (Laboratório de PROcessamento de Sinais, Aplicações Inteligentes e COmunicações www.prosaico.uerj.br) e atua principalmente nas áreas de processamento de sinais e comunicações. 